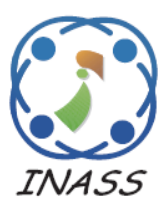

\title{
A Novel Adaptive Channel Allocation Scheme for Multi-Cell Massive MIMO Systems
}

\author{
Sandeep Dhanraj Bawage ${ }^{1 *}$ \\ Manjula Shivkumar ${ }^{1}$ \\ ${ }^{1}$ Department of Electronics \& Communication Engineering, Lingaraj Appa Engineering College, \\ Bidar, Karnataka, India \\ * Corresponding author's Email: sandeepbawage@gmail.com
}

\begin{abstract}
Massive Multi-In-Multi-Out (MIMO) is a promising and popular technique in wireless communication systems, which is used to deliver high reliability, spectral efficiency to several users. The major problem arises in channel contamination and allocation in a massive-MIMO network. In this paper, we proposed a novel adaptive channel allocation scheme (NACAS) to reduce channel contamination and improve channel allocation. The process of channel assignment is randomized at each time slot where data is repetitively transmitted with successive-interferencecancellation across similar packets. Massive-MIMO has the property of successive interference cancellation and majorly depends on asymptotic in-variance power which is received from the user in less time of interval, and asymptotic orthogonality among the user channels. Therefore, the proposed method permit received signals that turn contaminated into the combination of linear data, which provides an increment in throughput compared to the conventional techniques. The numeric and graphical analysis is shown in the result section, evaluation parameters are considered such as throughput, number of antennas, fading effect, etc. where our proposed approach has shown significant improvement as compared to state of art techniques.
\end{abstract}

Keywords: Multi-In-multi-out (MIMO), Wireless communication, Up-link, Channel allocation, Throughput.

\section{Introduction}

MIMO systems are widely utilized in the wireless-communication systems (WCSs) when spatial multiplexing can maximize the efficiency of spectral [1]. LAN (Local Area Network) and cellular systems are maximizing the number of antennas and it is estimated to maximize the demands for higher throughputs. Whereas the AP (access point) has an enormous number of the massive MIMO [2], precoding methods can maximize the throughput, and transmission power can minimize the CSI (Channelstate-Information) [3]. CSI plays an eminent role in the coherent-communication. In TDD (time-divisionduplex), MIMO-transmission, UL (Uplink) CSI at BS (Base station) can be evaluated from an orthogonal pilot, and the channel of DL (downlink) uses channel reciprocity [3]. The channel estimation has two reasons for the massive access scenarios [4]. Firstly, the devices are lower cost, the duration of channel allocation has a limited budget for UL power. Mobile devices are limited by coherence time.
Anyways, the number of devices is higher than the obtainable orthogonal pilots, and it is not possible for given devices that have dedicated pilots. Secondly, each device transmits the data to BS in an intermittent pattern. Furthermore, it is not required to assign dedicated pilots for all of the given devices within the network.

Since, deployed systems are in the mode of TDD rather than FDD (frequency-division-duplexing), getting CSI at BS, becomes one of the challenging issues. To address this problem, code-based methods and VQ (vector-quantization) have been introduced in [5]. Particularly, the size of the codebook becomes higher if higher CSI is chosen at BS. To minimize feedback-overhead, few methods are used channel correlation and statistics have been introduced. In paper [6], the channel extrapolation uses the correlations among UL and DL-CSI in the systems of FDD to minimize the feedback-overhead. In paper [7], the CSI of user statistical is utilized to design the statistical-3D-DL beamforming transmission method. Compared with the multi-user-MIMO transmission 
algorithm that needs an instantaneous-CSI at BS, this method can minimize feedback overhead and channel estimation. Anyways, an extrapolated channel utilizes statistical-CSI that can't obtain similar performance as the utilization of instantaneous-CSI from estimation errors.

The system of massive-MIMO has been introduced in $[8,9]$, in which there are tens of the users at the receive side which is served as hundreds of sending antennas. The standard pre-codes and identifications like MRC (maximal ratio combining), ZF (Zero Forcing) and MRT (maximal-ratio transmission) have been assumed in [10] to analyse the system performance of massive-MIMO. The scaling law and performance analysis have been introduced in [11] by utilizing MRT and MRC methods when massive-MIMO relay on the networks. Introduced in [12, 13], a novel method utilizing fullduplex-BS, the sequential beam-forming through closed-loop training and in-band to execute DT (datatransmission) with the acquisition of CSI. When consecutive creating more than one user pre-beam and pre-coder former [14] for maximizing sum-rate a novel beam-forming method is introduced which is using MMSE-method called JSDM (joint-spatial division-multiplexing). Many users are assisted by more than one antenna in multiuser-MIMO. The performance analysis has been done using gain among antenna-pair [15]. To eliminate the interference of inter-user on the UL side methods such as identification of maximum-likelihoodmultiuser are utilized in [16]. For communication among BS and users in various resources of timefrequency, a channel is orthogonal for the point of information-theoretic, which is not favourable in this channel. So the communication should take place in similar resources of time-frequency [17] to obtain higher rates. Nowadays, BS is placed with a higher number of antennas in multi-user MIMO. In paper [16], the analysis of selective decode -the forward cooperative network has been introduced. An interference of intra-cell can be minimized by using the processing of normal signal [17] in higher arrays. The analysis of time selective has been done utilizing the nakagami-m channel for the MN (mobile node) and it can be represented the performance of the static system which is much better than the mobile system [18].

This paper follows the procedure of ACA (Adaptive-Channel-Allocation). The terminal sends probability and transmits the channel in UL by data part. The channel assignment can be randomized in every single time slot while the data part is repetitive, SIC across similar packets. The implementation of SIC (successive-interference-cancellation) is a new property of massive MIMO, as it relies on 2 different specific contexts like asymptotic in-variance power which is received from the user in less time of interval, and asymptotic orthogonality among the user channels. These properties permit received signals that turn contaminated into the combination of linear data. These linear combinations form code-word that can be decoded by utilizing SIC, which gives maximization in throughput compared to the conventional techniques. Anyways transmission probability can be optimized for free contamination of transmission when SIC isn't available in massiveMIMO.

This study proposed a novel adaptive channel allocation scheme for the system of massive-MIMO and provided complete elaboration on the tree analysis of ACA. In a different way, this assumes channel-code at physical-layer that exists in the practical systems, which causes the performance and design of ACA. The performance of the proposed PPA approach is evaluated in terms of the throughput uplink rate and the results are compared with other schemes.

This paper is organized in such a way that section-2 represents the previous paper related to massive-MIMO and section-3 represents the proposed methodology. Section 4 provides the numerical and graphical analysis of results and, our work is concluded in section 5 .

\section{Literature survey}

Many techniques related to existing methods are represented as follows. In paper [19], the author represented an approach of channel scheduling based on the user grouping to address issues of channel contamination. The degradation of a user is calculated for allocating the sequence of an optimal channel to users, who greedily suffered the degradation. This method minimized the effects of shadowing fading based on sum-rates and improvised the system performance, but higher overhead causes in pilots. In paper [20], represented the multi-cell multi-user MIMO-system based on spectral efficiency and online beamforming. In this case, lower and upper bound was used to obtain the effective rate of a downlink. The ZF and MRT beamforming were used in a massive-MIMO system for obtaining a closed-form of an expression. Here, the performance was discovered better, but having massive-pilot overhead. In the paper [21], the author modeled the BDF function, and the wiener-based filter is based on the performance of the per-cell rate and error of channel estimation for conquering the effects of pilot contamination. 
In the paper [22], simple-2-ADC (advancedconverters) pulled into consideration a larger number of MIMO and frameworks with a high capacity of data transfer. Mainly, 1-bit of ADCs are suitable for such type of framework due to their lower power usage and cost. They delineate the benefits of image rate that examining in the UL-monstrous-MIMO system with the help of 1-bit of ADCs in SER. They define the FTSR provides around 5-dB to SNR benefits in terms of the SER. In paper [23], introduced channel estimation that can be utilized to find quality systems of MU-MIMO as refreshing the method of channel assessment that uses the symbols of reliable soft. They gather reliable information tones from interfering and desired users related to the conclusion and relate them as pilots for channel evaluation. The data decoding of channel assessment is achieved iteratively when data and channel symbols are integrated.

In paper [24], the author assumed $\mathrm{CE}$ issue for the system of mm-wave with RF-chain and utilized at MS and BS. Various existing studies are investigated narrowband channels, they assumed the estimation of wideband channels with mm-wave with frequency selectivity, containing fading coefficients and time delays. Two phases compacted the sensing algorithm with a lower rank matrix that permits the sampling process to be carried out. In the paper [25], the author investigated the effect of channel ER (estimationerror) on capacity-MIMO fading-channels. They study upper and lower bounds of mutual data within the channel-estimation error and represent 2 bounds are very tight for the Gaussian inputs. Considering Gaussian inputs, they develop lower bounds of outage capacities, ergodic, and the optimal transmitter-power allocation methods obtain bounds within the perfect-feedback. The optimal method can be modified water-filling over fading and antenna domains. This method is close to the optimum within smaller feedback delays but a delay is larger and similar powers should be assigned across spatial dimensions. Furthermore, the computational complexity of space-time water-filling is lower than that of spatial water-filling. It's also proven that space-time water-filling necessitates previous knowledge of the channel gain distribution, and that the spectral efficiency advantage over spatial waterfilling comes at the cost of increased channel outage probability for Rayleigh channels with log-normal shadowing. Whereas in paper [26], they estimated various performances of water-filling algorithms. They have chosen 4 different AIWF (Adaptiveiterative water-filling), PA (power-allocation), CWF (conventional water-filling), and IWF (inverse-water filling) algorithms. Capacity is one of the performance metrics, they utilized to compare the metric by taking power allocation of optimality transmission.

The PA (power-allocation) can be computed with the reference value of water level has various methods for various algorithms. Hence, outcomes represent the AIWF algorithm that has a good effect on the MIMO-OFDM system by assigning the power. The author assumes channel allocation, integrated beam-forcing, and power by utilizing the algorithm of simulation annealing and GA (genetic-algorithm) in MIMO-CRN (Cognitive radio-networks). In this paper [27], a cognitive network has considered, where the spectrum of primary users can be utilized by secondary-user transmitters to increase the utilization of spectrum while the interference of intrauser is reduced by implementing the beam-forcing at every single SU-TX. After, formulate the process on $\mathrm{CRN}$, they implement the structure of beam-forcing on the SVD. Because there are additional power limits in a cognitive radio system, a cognitive network cannot be used directly for power allocation. In paper [28], the author assumes DL and UL of noncooperative multi-cellular-TDD methods, considering the number of antennas per BS and the number of user terminals per cell is larger. Their system accounts for pilot contamination and channel estimation, correlation of antenna, and arbitrary path loss for every single link, which cause for computational complexity.

\section{Optimized cluster establishment and cluster-head Selection}

Here, we indicate metrics in the bold upper case, scalars in the lower-case, and vectors in the bold lower case. The superscripts indicate the transpose as $\mathbb{T}$ and the superscript $*$ simplifies the complex conjugate and the super-script $\mathcal{H}$ simplifies as the transpose of the conjugate.

We assume RAS (Random access system) containing only one BS (Base station) with users $\mathcal{U}$ and antennas $\mathcal{A}$ for each BS with only one antenna. The communication is carried out by utilizing slottedtime, where every single time-slot contains the ULchannel stage, DL data, and UL data stage. Fig. 1 depicts a block diagram of massive-MIMO. The below figure represents a block diagram of massiveMIMO, which contains BS, the number of the user terminals, and antenna arrays. BS sends data from various antennas into different terminals at a similar frequency. The massive-MIMO is used to deliver improvised coverage and higher EE (energyefficiency), higher rates, link-reliability, but in the case of massive-MIMO faces many challenges for 


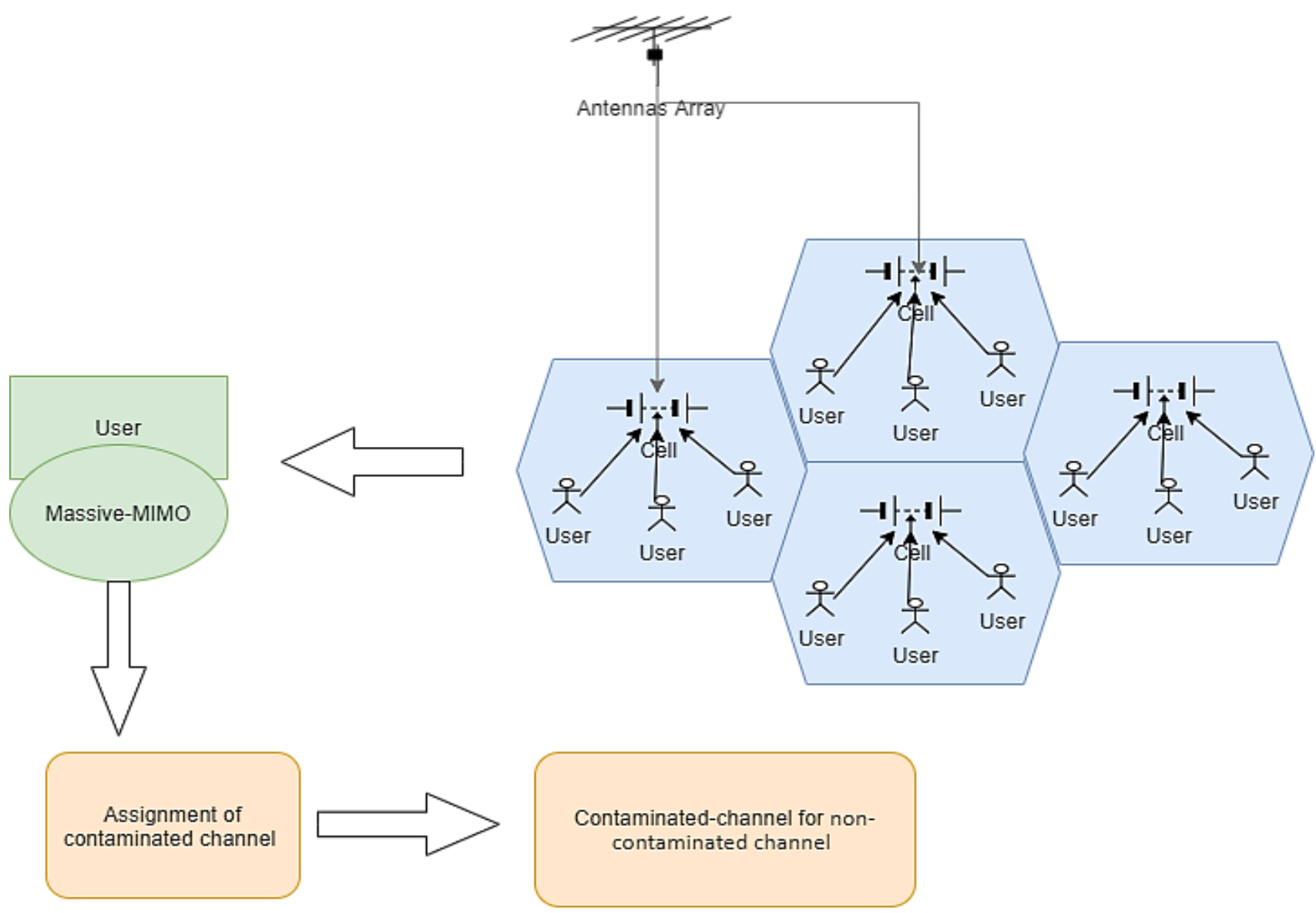

Figure. 1 Sensor-node and cluster-based WSN

channel allocation and channel estimation, which minimizes the system performance of massiveMIMO.

Therefore, a novel technique is proposed for mitigating the channel allocation to solve the problems of a channel. In every single time slot, every single user is active with the help of probability $P_{x}$. There are $t$ orthogonal channel sequences $\{\mathbb{S}\}$, each sequence containing $t$ symbols $\mathbb{S}=$ $[\mathbb{s}(1) \mathbb{s}(2) \ldots . \mathbb{s}(t)]$. The alive user chooses channel sequence through an available channel sequence $t$. More than one user can select a similar channel sequence. The channel among $u$ th user and BS during nth time-slot is described as $k_{n, u}=$ $\left[k_{n, u}(1) k_{n, u}(2) \ldots . k_{n, u}(\mathcal{A})^{\mathbb{T}}\right]$, where $k_{n, u} \forall n, u$ are i.i.d. Afterward, we assume systems that apply the ideal control of power, such as $k_{n, u}(a) \sim C N(0,1), \forall a^{1}$. The time slot has a limited duration $\mathbb{T}_{c}$, that corresponds to coherence-time in which the coefficient of the channel remains const. Let, $\mathbb{A}_{n}$ is the active user in the $n$ time-slot, while $\boldsymbol{B}_{n}^{b}$ is the set of users that have chosen $\mathbb{S}_{b}$ in $n$th timeslot. If $B_{n}^{P r}$ represents uplink channel signal that received in the time-slot $n$, we have;

$$
\boldsymbol{B}_{n}^{p r}=\sum_{b=1}^{\mathrm{t}} \sum_{k \in \mathbb{A}_{n}^{b}} \boldsymbol{k}_{u, n} \mathbb{S}_{b}+\boldsymbol{C}_{n}^{p r}
$$

Where, $\boldsymbol{C}_{n}^{p r}$ is the i.i.d matrix. The components of Gaussian noise $\boldsymbol{C}_{n}^{p r}(a, b) \sim C N\left(0, \sigma_{n}^{2}\right), \forall_{a, b}$. The instance of future vector $c$ or the matrix $\boldsymbol{C}$ with various sub or super-scripts follow a similar definition. All alive users send the message of length $L_{r}$ in the stage of UL data. The message through $k t h$ user symbolized $a_{u}^{r}=\left[a_{u}^{r}(1) a_{u}^{r}(2) \ldots \ldots a_{u}^{r}\left(L_{r}\right)\right]$. Utilizing $\boldsymbol{B}_{n}^{r} \in Z^{\mathcal{A} \times L_{r}}$, to symbolize data part of the received signal in the uplink, we obtain:

$$
\boldsymbol{B}_{n}^{r}=\sum_{k \in X_{n}^{b}} \boldsymbol{k}_{n, u} \boldsymbol{a}_{j}+\boldsymbol{C}_{n}^{r}
$$

In the phase of downlink, we send channel reciprocity, such as the channel of UL is considered to be the valid estimation for the transmission of DL. The base station sends pre-coded DL channel symbol, 
such as $n-t h$ user gets DL channel signal, $b_{n, u}^{r d}$, given by;

$$
b_{n, u}^{p d}=\boldsymbol{k}_{n, u}^{T} \boldsymbol{w}_{n, u}+\boldsymbol{c}_{n, u}^{p d}
$$

Where, $\boldsymbol{w}_{n, u}=$ $\left[w_{n, u}(1) w_{n, u}(2) \ldots . . w_{n, u}(A)^{T}=\boldsymbol{k}_{n, u}^{*}\right.$ is denoted as the precoding vector for the user $u$ in the $\mathrm{n}$-th timeslot. This considers the BS estimates through the UL channel $\boldsymbol{k}_{n, u}$ before the transmission of DL. We symbolize DL message for the user $u, a_{u}^{d}=$ $\left[a_{u}^{d}(1) a_{u}^{d}(2) \ldots a_{u}^{d}\left(L_{d}\right)\right]$ and describe $\boldsymbol{B}^{d}$ with the help of $u$ th row that is given by $\boldsymbol{a}_{u}^{d}$ for $u \in Z_{n}$. Additionally, we describe $\mathcal{W}_{n}$ with $u$ - th column that being $\boldsymbol{w}_{n, u}$ and $\mathbb{H}_{n}$ with $\boldsymbol{k}_{n, u}$ as a u-th column for $u \in Z_{n}$. The signal of DL data is received by:

$$
\boldsymbol{B}_{n}^{d}=\mathbb{H}_{n}^{T} \mathcal{W}_{n} \boldsymbol{A}^{d}+\boldsymbol{C}_{n}^{d}
$$

In both DL and UL, coherence time permits $L$ symbols of transmission and we have $D=t+L=$ $t+L_{r}+L_{d} . L_{r}$ and $L_{d}$ is defined as selected arbitrarily for the operation of asymmetric as long as $L=D-t$, we assume $L_{r}=L_{d}=0.5 L$. Data is considered as the channel coded concerning rate $R$ at the PHY layer, such as the rate of effective data is $\mathbb{R} \frac{L}{2 D}$. We assume the codes of an arbitrary channel with hard identification decoding and apply UB (upper-bound) on the capabilities of error correction codes.

Precisely, we assume the data message is recovered if $p_{e} \leq \frac{(1-\mathbb{R})}{2}$, where $p_{e}$ is BER (bit-errorrate). Hence, the outcomes of numerical serve as the UBs (upper-bounds) through the perspective of channel code. With the help of $\left|\mathbb{S}_{n}\right| \leq t$ and $\left|\mathbb{S}_{n}\right| \leq$ $\left|A_{n}\right|$. The system throughput is described as sum rate in the time slot $n, y_{n}$.

$$
y_{n}=\frac{\left|\mathbb{S}_{n}\right| \mathbb{R}(D-t)}{2 D}=\frac{\left|\mathbb{S}_{n}\right| \mathbb{R} L}{2 D}
$$

In time slot, $y_{n}$ is described as the recovered messages. The rate of modulation can be selected arbitrarily and it will influence the distribution of probability $\left|\mathbb{S}_{n}\right|$ and throughput.

\section{Novel Adaptive Channel Allocation}

In this allocation, standard methods of communication are treated as UL operation.

The transmission is structured in a consecutive block of time slots $\Delta$, which is referred to as a frame. If any user is alive more than one time with the help of a frame, UL data is presented for conventional coded of RAS (Random-Access-Schemas). We propose a parameter known as the overhead factor $\propto$, described as;

$$
\propto=\frac{t \Delta}{U}
$$

In a frame, a normalized expression is defined as the orthogonal resources. Ideally, $\propto=1$ is an orthogonal resource per-user. Anyway, the scheme needs smaller overhead to operate well at the length of the finite-frame. $\propto$ will achieve higher values. The interest of performance parameter has an average UL frame throughput which is given by $y_{r}=\sum_{n=1}^{\Delta} y_{n} / \Delta$.

From UL channel signals in Eq. (1), it is possible to evaluate the channels among BS and users. Anyways, more than one user may apply a similar channel sequence, it is possible to evaluate the sum of convoluted channels. In order to evaluate the least squares, $\phi_{n, b}$ is basically based on the channel signal in the time slot $n$ through the users applying $s_{j}$ is discovered as;

$$
\begin{aligned}
\phi_{n, b} & =\left(\mathbb{S}_{b} \mathbb{S}_{b}^{\mathcal{H}}\right)^{-1} \boldsymbol{B}_{n}^{p r} \mathbb{s}_{j}^{\mathcal{H}} \\
& =\sum_{u \in \mathcal{A}_{n}^{b}} \boldsymbol{k}_{n, u}+\boldsymbol{c}_{n}^{\text {pr }}
\end{aligned}
$$

Where, $\boldsymbol{c}_{n}^{p r^{\prime}}$ is defined as aa processed noise which is originating from $\boldsymbol{c}_{n}^{p r}$. The upcoming vector instances $z$ with the prime follows a similar definition.

The issue of interfering users applying similar or non-orthogonal, channel sequences is known as channel contamination. If we ensure to identify the data in the UL stage by utilizing the contaminated channel, an outcome will be the summation of the data messages. By $\Psi_{n, b}$, we describe data in order to evaluate $\phi_{n, j}$. Considering orthogonality among user channels such as $\frac{\lim _{A \rightarrow \infty} k_{n, m}^{\mathcal{H}} k_{n, u}}{A}=0$ for $m \neq u$. We have,

$$
\begin{aligned}
\boldsymbol{\Psi}_{n, b} & =\left(\boldsymbol{\phi}_{n, b}^{\mathcal{H}} \boldsymbol{\phi}_{n, b}\right)^{-1} \boldsymbol{\phi}_{n, b}^{\mathcal{H}} \boldsymbol{B}_{n}^{r} \\
& =\sum_{u \in \mathcal{A}_{n}^{j}} \frac{\left(\boldsymbol{\phi}_{n, j}^{H} h_{n, k}\right)}{\left\|\boldsymbol{\phi}_{n, j}\right\|^{2}} \boldsymbol{a}_{u}^{r}+\mathbf{z}_{n}^{r^{\prime}}
\end{aligned}
$$

Hence, channel collision leading to data collision such as interference between the data signals. The classical way is to deal with this issue, this collision is to reduce the contamination probability by 
choosing $p_{x}$. The main objective criterion is to reduce the probability of having one user that applying on particular channel sequence in the particular time-slot. Hence, we have,

$$
\begin{aligned}
& \text { maximize } \quad \mathcal{P}_{x} \operatorname{Pr}\left(\left|\mathcal{A}_{n}^{b}\right|=1\right) \\
& \text { subject } \quad 0 \leq p_{x} \leq 1
\end{aligned}
$$

This will exploit the number of the noncontaminated channel which estimates to increase the number of successful data transmissions.

A new solution is represented that doesn't assume the data collisions but collided signals and utilize them from the iterative process, whereas they denote the throughput. We call it coded channel access (CCA). This is based on the contaminated that evaluates as matched filters on received UL datasignals, $\boldsymbol{B}_{n}^{r}$. The signal of filter data is defined as $\mathfrak{f}_{n, b}=\in \boldsymbol{Z}^{1 \times L_{r}}$,

$$
\begin{gathered}
\mathfrak{f}_{n, b}=\boldsymbol{\phi}_{n, b}^{\mathcal{H}} \boldsymbol{B}_{n}^{r} \\
=\sum_{\substack{u \in \mathcal{A}_{n}^{b}\\
}}\left(\left\|\boldsymbol{k}_{n, u}\right\|^{2}+\sum_{\substack{m \in \mathcal{A}_{n}^{b} \backslash\{u\}\\
}} \boldsymbol{k}_{n, m}^{\mathcal{H}} \boldsymbol{k}_{n, u}\right) a_{u}^{r} \\
\\
\sum_{l \in \mathcal{A}^{b} \backslash \mathcal{A}_{n}^{b}}\left(\sum_{o \in \mathcal{A}_{n}^{b}} \boldsymbol{k}_{n, o}^{\mathcal{H}} \boldsymbol{k}_{n, l}\right) \boldsymbol{a}_{l}^{r}+c_{n}^{r^{\prime}}
\end{gathered}
$$

Compared to the identification in Eq. (8), we don't normalize the estimation of channel power. The normalization will give data messages in a collision. The signals of filtered data contain more than one data message and the power of the channel contains more than one user channel, whereas the normalization is known as futile. Now, we end the identification after matched the filtering phase and buffer signals. By sending on 2 essential structures from the scenario of massive-MIMO, Eq. (10) can be simplified when $M$ goes towards infinity. This implies orthogonality among the vectors of userchannel. This implies $\frac{\lim _{A \rightarrow \infty} k_{n, m}^{\mathcal{H}} k_{n, u}}{A}=0$ for $m \neq$ $u .2^{\text {nd }}$ feature is the stability of temporal-channel powers, which implies that $\left\|\boldsymbol{k}_{n, u}\right\|^{2}=$ $\left\|\boldsymbol{k}_{n^{\prime}, u}\right\|^{2} \forall n, n^{\prime}$. This permits us to drip the index of time in channel-powers. We follow an expression for the signal of filtered data in $M \rightarrow \infty$ limit.

$$
\lim _{A \rightarrow \infty} \mathfrak{f}_{n, b}=\sum_{k \in \mathcal{A}_{n}^{j}}\left\|\boldsymbol{k}_{u}\right\|^{2} \boldsymbol{a}_{u}^{r}+\boldsymbol{c}_{n}^{r^{\prime}}
$$

Hence, the implication of the channel contamination has turned into the linear combinations of the data-messages, from postprocessing with the matched filters. The combination of linear coefficients is the power of a stable channel. By relying on the properties of massive-MIMO asymptotic, the channel vector evaluates in Eq. (7) that can be used to discover the sum of channelpowers. We define $\boldsymbol{g}_{n, b}$ as:

$$
\begin{gathered}
\boldsymbol{g}_{n, b}=\boldsymbol{\phi}_{n, b}^{\mathcal{H}} \boldsymbol{\phi}_{n, b} \\
\sum_{k \in \mathcal{A}_{n}^{j}}\left(\left\|\boldsymbol{k}_{n, u}\right\|^{2}+\sum_{m \in \mathcal{A}_{n \backslash\{u\}}^{j}} \boldsymbol{k}_{n, m}^{\mathcal{H}} \boldsymbol{k}_{n, u}\right)+c_{n}^{p r^{\prime}} \\
\lim _{A \rightarrow \infty} \boldsymbol{g}_{n, b}=\sum_{k \in \mathcal{A}_{n}^{j}}\left\|\boldsymbol{k}_{u}\right\|^{2}+\boldsymbol{c}_{n}^{r^{\prime}}
\end{gathered}
$$

In Eqs. (11) and (12) for $n=1, \ldots, \Delta$, and $b-$ $1 \ldots, t$, shows the equation systems, in order to resolve for $\boldsymbol{a}_{u}^{r}, k=1, \ldots, U$. Note that, BS has no previous knowledge of channel choices and random activity of users. Hence, the equation system can-not be resolved by utilizing Gaussian-elimination.

SIC describes as follows. Firstly, BS discovers decodable UL-data such as $\left|\mathcal{A}_{n}^{b}\right|=1$. If $\mathfrak{f}_{n, b}$ is defined as decodable, we have to evaluate the ULdata that embeds data about data transmission and random channel of transmitter schedule, which permits BS to place all replicas of a similar packet transmitter. Anyways, BS has no previous knowledge of channel choices, it's available before the cancellation of interference. In Eqs. (11) and (12), when information from the user $u$ is decoded successfully, BS studies $b$ and $n$ we have $u \in \mathcal{A}_{n}^{j}$. This allows BS to cancel an interference because of replicas from the user $u$ which is subtracting $\left\|\boldsymbol{k}_{n, u}\right\|^{2} a_{u}^{r}$ from $\boldsymbol{f}_{n, b}$ for $u \in \mathcal{A}_{n}^{b}$. Moreover, an interference caused by the help of an associated channel transmission can be canceled by $\mathcal{A}_{n}^{b}$. This allows BS to cancel an interference because of replicas from the user $u$ which is subtracting $\left\|\boldsymbol{k}_{n}\right\|^{2}$ from $\boldsymbol{g}_{n, b}$ for $u \in \mathcal{A}_{n}^{b}$. The cancellation $u$ is eliminated from $\mathcal{A}_{n}^{b}$. it originally performed. This leads to novel cases $\left|\mathcal{A}_{n}^{b}.\right|=1$, whereas novel data can be eliminated and continued an iterative process. The data operation of UL is defined in Algo-1.

The utilized decoding algorithm is analogous to BP (belief propagation) decoding of erasure codes. the common way is visualizing such codes as bipartite graphs.

The performance of BP-decoder for erasure code is interconnected with variable and factor nodedegree distribution, described as $\Lambda$ and $\psi$ where 
$\psi_{d} / \wedge_{d}$ is the probability and the variable/factor node has the degree $d$. Many works have been studied for performing degree-distributions.

$$
\begin{gathered}
\psi_{d}=\operatorname{Pr}\left(\left|\mathcal{A}_{n}^{b}\right|=d\right)=\left(\frac{U}{d}\right)\left(\frac{p_{x}}{t}\right)^{d}\left(\frac{p_{x}}{t}\right)^{u-d} \\
\approx \frac{\left(\frac{p_{x} u}{t}\right)^{d}}{d !} e^{-\frac{p_{x} k}{t}=\frac{\beta^{d}}{d !} e^{-\beta}}
\end{gathered}
$$
node.

Where $\beta$ is defined the average degree of factor

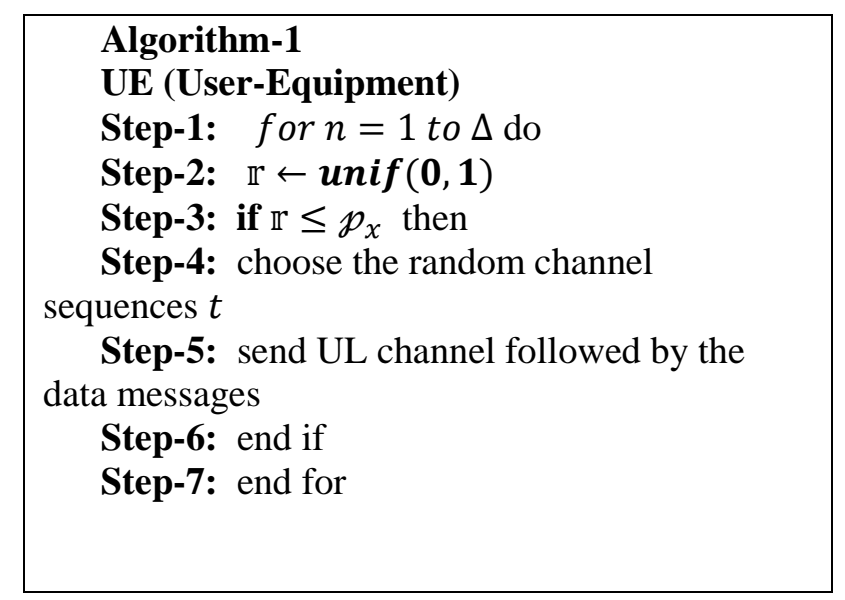

\section{BS (Base Station)}

Step-1: buffer $\leftarrow \varnothing$

Step-2: for $n=1$ to $\Delta$ do

Step-3: Receive $B_{n}^{p r}$ and $B_{n}^{r}$.

Step-4: $\quad$ for $b=1$ to $\mathrm{t}$ do

Step-5: $\quad \phi_{n, b} \leftarrow\left(\mathbb{s}_{b} \mathbb{s}_{b}^{\mathcal{H}}\right)^{-1} \boldsymbol{B}_{n}^{p r} \mathbb{s}_{j}^{\mathcal{H}}$

Step-6: $\quad \boldsymbol{f}_{n, b} \leftarrow \boldsymbol{\phi}_{n, b}^{\mathcal{H}} \boldsymbol{B}_{n}^{r}$

Step-7: $\quad \boldsymbol{g}_{n, b} \leftarrow \boldsymbol{\phi}_{n, b}^{\mathcal{H}} \boldsymbol{\phi}_{n, b}$

Step-8: if decodable is $\frac{f_{n, b}}{g_{n, b}}$ at the layer of physical then

Step-9: Include $\boldsymbol{a}_{n}^{r}$ and $\left\|\boldsymbol{k}_{u}\right\|^{2}=\boldsymbol{g}_{n, b}$ to the buffer.

Step-10: end if

Step-11: end for

Step-12: end for

Step-13: while buffer $\neq \emptyset$

Step-14: Access buffer data $\boldsymbol{a}_{n}^{r}$

Step-15: Access the norm of buffer channel $\left\|\widehat{\mathfrak{k}}_{u}\right\|^{2}$

Step-16: Remove the pattern of channel activity of $r$ user which is embedded in $\boldsymbol{a}_{n}^{r}$

Step-17: $\quad$ for $n=1$ to $\Delta$ do

Step-18: $\quad$ for $b=1$ to $t$ do

Step-19: $\quad$ if $\boldsymbol{u} \in \mathcal{A}_{n}^{b}$ then

\begin{tabular}{|c|c|}
\hline Step-20: & $\boldsymbol{f}_{n, b} \leftarrow \mathfrak{f}_{n, b}-\left\|\widehat{\mathfrak{k}}_{u}\right\|^{2} \boldsymbol{a}_{n}^{r}$ \\
Step-21: $\quad \quad \boldsymbol{g}_{n, b} \leftarrow \boldsymbol{g}_{n, b}-\left\|\widehat{\mathfrak{k}}_{u}\right\|^{2}$ \\
Step-22: $\quad$ if decodable is $\frac{\mathfrak{b}_{n, b}}{g_{n, b}}$ at the \\
layer of physical then \\
Step-23: $\quad$ Include $\boldsymbol{a}_{n}^{r}$ and $\left\|\widehat{\mathfrak{k}}_{u}\right\|^{2}=$ \\
$\boldsymbol{g}_{n, b}$ to the buffer. \\
Step-24: $\quad$ end if \\
Step-25: & end if \\
Step-26: & end for \\
Step-27: & end for \\
Step-28: & end while \\
\hline
\end{tabular}

$$
\beta=\frac{p_{x} u}{t}
$$

and

$$
\begin{aligned}
\Lambda_{d} & =\left(\begin{array}{c}
\triangle \\
d
\end{array}\right) p_{x}^{d}\left(1-p_{x}\right)^{\triangle-d} \approx \frac{\left(\triangle p_{x}\right)}{d !} e^{-\triangle p_{x}} \\
& =\frac{(\propto \beta)^{d}}{d !} e^{-\propto \beta}
\end{aligned}
$$

Where

$$
\propto=\frac{t \Delta}{K}
$$

Anyway, we don't have full freedom to the distributions of degree. The distributions of an influencing degree are an overhead factor $\propto$ and $p_{x}$ Eq. (6). Since the user is applying the channel sequence in the time slot with probability $p_{x} / t$ and there are time slots $\Delta$, we have following relation among the degree-distributions and $p_{x}$ and $\propto$ in Eq. (13). Through the choice of $\propto$ and $\beta$, among them, one defines the degree distributions.

\section{Result and analysis}

This section consists of the description of our experimental setup and performance analysis with state-of-art techniques. The system configuration, 12GB of RAM, and Intel i5 Processor with Windows 10 operating system. In the experimental setup, we have used the MatLab tool for the complete simulation of the massive MIMO system. However, the communication between the BS and user is prepared through transmitting the pilot's signal to calculate the channels, where the user may communicate with the pilots at the same time that causes the channel contamination. Here, we have proposed NACAS that can able to provide optimal 
channel allocation and reduce channel contamination in terms of obtained throughput.

The Weighted-Graph-Coloring-Based Pilot Decontamination (WGC-PD) greedily allocates different pilots to associated users with a huge weight in edge-weighted interference graph [29]. In practical mobile WSN of long-term development systems, WGC-PD may cause the exhaustive search resolution to multiple optimization problems is usually infeasible due to its exponential cumulative property and result computational complexity. Here, we presented a resolution based on coded RAS to pilot sequences that influences on the channel habituation properties of massive-MIMO. In addition, these allow to view a contaminated pilot signals set as the graph code, where BP is performed to optimize channel estimation process.

The performance analysis of our developed scheme is done by varying the number of antennas, log-normal shadowing fading, transmission power, and number of users. In addition, comparative techniques such as MRT-ZF [20], WGC-PD [29], and DPSM (degradation-based pilot scheduling method) [19] are used for the assessment with the proposed approach NACAS for the analysis.

Here, we performed multiple simulations, where a typical hexagonal cellular network with 10 and 20 cells is considered. Each cell has several users with a single antenna, antennas, and BS, center cell surrounded via the various other cells that are considered to be target cell. The system parameter is briefly given in Table 1.

Figs. 2 and 3 shows the impact of the number of BS antennas on the achievable throughput at cell-10 and cell-20, where BS antennas vary from 10 to 5000, cell radius is $500 \mathrm{~m}$, path loss 3.8 , number of users in each cell is 10 , spectral efficiency 0.1 , the data and pilot transmission power are $15 \mathrm{~dB}$ and $\log$-normal

Table 1. Basic parameters of network simulation

\begin{tabular}{|l|l|}
\hline Number of cells & 10,20 \\
\hline Number of BS antennas & $10-5000$ \\
\hline Cell radius & $500 \mathrm{~m}$ \\
\hline Path loss & 3.8 \\
\hline $\begin{array}{l}\text { Number of users in each } \\
\text { cell }\end{array}$ & 10 \\
\hline Spectral efficiency & 0.1 \\
\hline $\begin{array}{l}\text { The data transmission } \\
\text { power }\end{array}$ & $15 \mathrm{~dB}$ \\
\hline $\begin{array}{l}\text { The pilot transmission } \\
\text { power }\end{array}$ & $15 \mathrm{~dB}$ \\
\hline $\begin{array}{l}\text { Log normal shadowing } \\
\text { fading }\end{array}$ & $1-8 \mathrm{~dB}$ \\
\hline
\end{tabular}

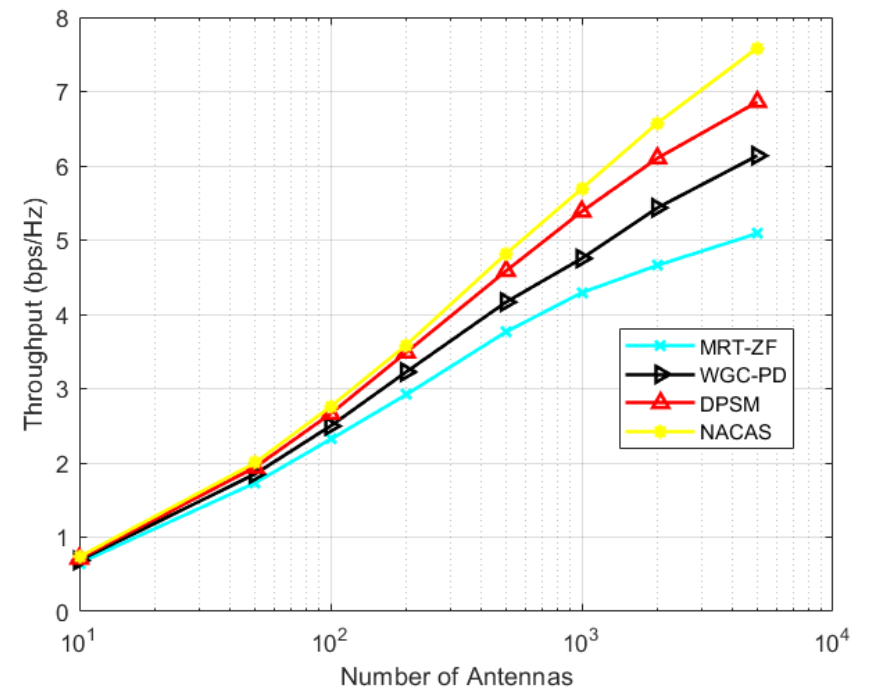

Figure. 1 Impact of the number of BS antennas on the achievable throughput (cell-10)

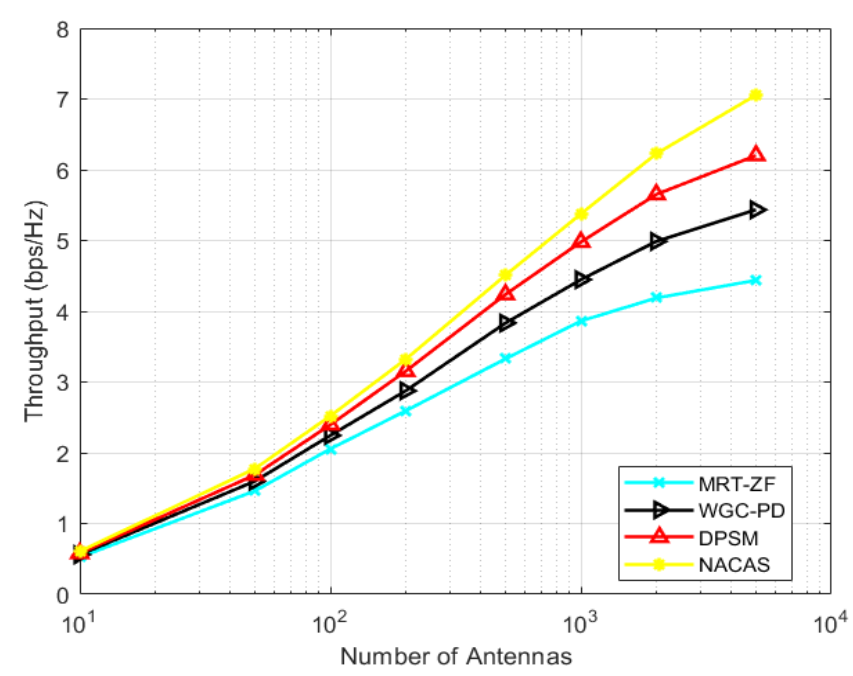

Figure 2 Impact of the number of BS antennas on the achievable throughput (cell-20)

shadowing fading is $8 \mathrm{~dB}$. In figure 2 , throughput at 500 BS antennas by the proposed method is 4.8 $\mathrm{bps} / \mathrm{Hz}$, which is $21.82 \%, 13.51 \%$, and $4.79 \%$ more compared to MRT-ZF, WGC-PD, and DPSM. Similarly, in Fig. 3 we can observe our proposed method performs a well-considering range of $\mathrm{BS}$ antennas, so it is obvious that our proposed scheme outperforms the state-of-art techniques. Table 2 shows the detailed comparative discussion of throughput based on the number of BS antennas.

Fig. 4 shows the impact of the log-normal shadowing fading on the achievable throughput at cell-10, where log-normal shadowing fading varies from 1 to $8 \mathrm{~dB}$, cell radius is $500 \mathrm{~m}$, path loss 3.8 , number of users in each cell is 10 , spectral efficiency 0.1 , the data and pilot transmission power is $15 \mathrm{~dB}$ and BS antennas are 512. In figure 4, throughput at 
Table 2. Comparative discussion based on number of BS antennas

\begin{tabular}{|c|c|c|c|c|}
\hline $\begin{array}{c}\text { No. of } \\
\text { Antennas }\end{array}$ & 10 & 50 & 100 & 200 \\
\hline \multirow[t]{4}{*}{ Cell-10 } & 0.651251 & 1.729819 & 2.318213 & 2.918925 \\
\hline & 0.686529 & 1.849876 & 2.496828 & 3.224073 \\
\hline & 0.71199 & 1.93902 & 2.661757 & 3.486924 \\
\hline & 0.735585 & 2.000365 & 2.756354 & 3.586464 \\
\hline \multirow[t]{4}{*}{ Cell-20 } & 0.524261 & 1.460975 & 2.054588 & 2.593025 \\
\hline & 0.559815 & 1.598333 & 2.245208 & 2.875691 \\
\hline & 0.579414 & 1.6864 & 2.395484 & 3.153709 \\
\hline & 0.607651 & 1.774574 & 2.520275 & 3.321827 \\
\hline $\begin{array}{c}\text { No. of } \\
\text { Antennas }\end{array}$ & 500 & 1000 & 2000 & 5000 \\
\hline \multirow[t]{4}{*}{ Cell-10 } & 3.765652 & 4.292739 & 4.660605 & 5.09004 \\
\hline & 4.165968 & 4.752453 & 5.434564 & 6.136686 \\
\hline & 4.585761 & 5.385303 & 6.102446 & 6.860347 \\
\hline & 4.816847 & 5.693593 & 6.575952 & 7.588336 \\
\hline \multirow[t]{4}{*}{ Cell-20 } & 3.327902 & 3.862211 & 4.187455 & 4.437246 \\
\hline & 3.832992 & 4.44689 & 4.987413 & 5.434612 \\
\hline & 4.237669 & 4.979045 & 5.651598 & 6.201402 \\
\hline & 4.508912 & 5.376219 & 6.226144 & 7.055695 \\
\hline
\end{tabular}

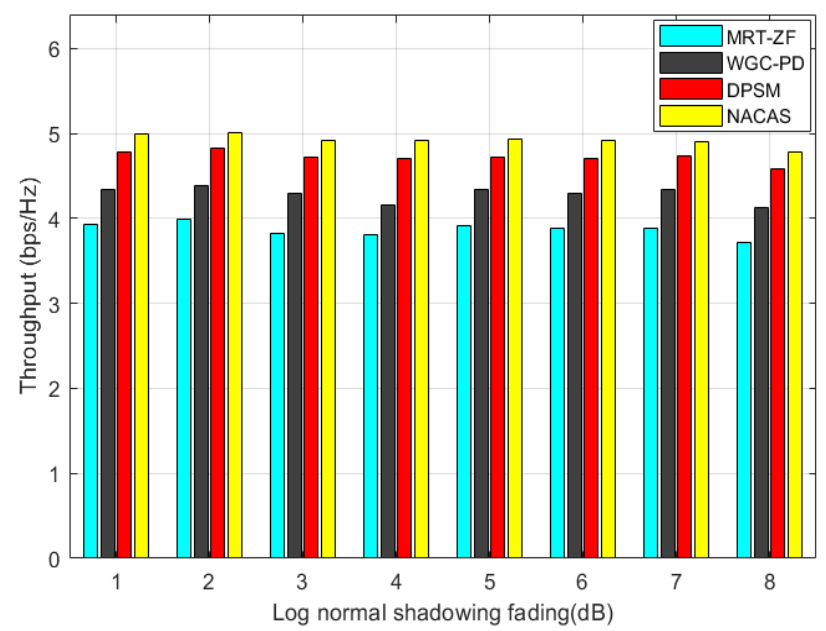

Figure. 3 Impact of the log normal shadowing fading on the achievable throughput (cell-10)

$8 \mathrm{~dB}$ log-normal shadowing fading antennas by the proposed method is $4.78 \mathrm{bps} / \mathrm{Hz}$, which is $22.47 \%$, $13.89 \%$, and $4.19 \%$ more compared to MRT-ZF, WGC-PD and DPSM. Similarly, figure 5 shows the impact of the log-normal shadowing fading on the achievable throughput at cell-20, where at $8 \mathrm{~dB} \log$ normal shadowing fading antennas by the proposed method is $4.54 \mathrm{bps} / \mathrm{Hz}$, which is $26 \%, 15 \%$, and $6 \%$ more compared to MRT-ZF, WGC-PD, and DPSM.

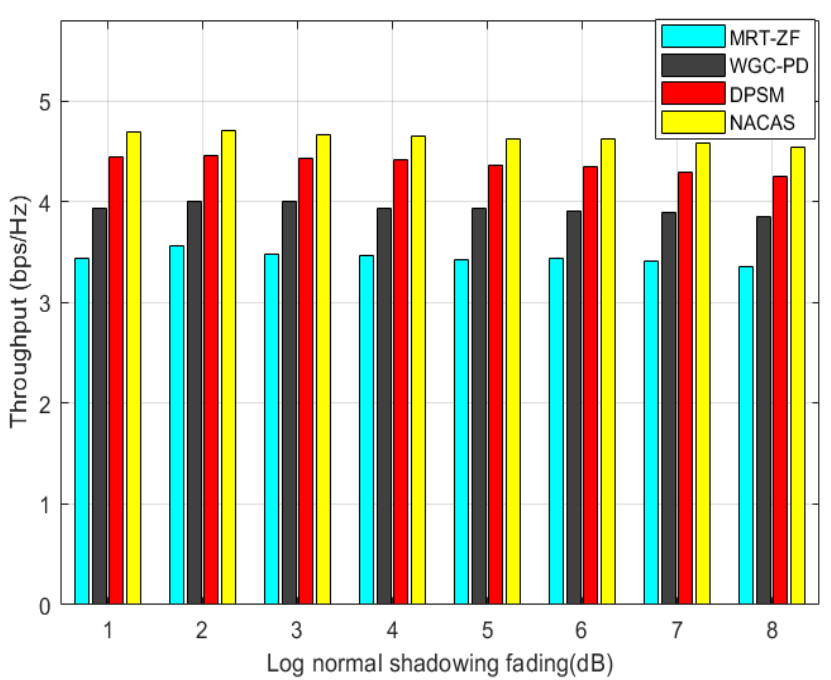

Figure. 4 Impact of the log normal shadowing fading on the achievable throughput (cell-20)

Table 3. Comparative discussion based on log normal shadowing fading

\begin{tabular}{|l|l|l|l|l|}
\hline Fading & 1 & 2 & 3 & 4 \\
\hline Cell-10 & 3.92635 & 3.99022 & 3.830215 & 3.803162 \\
\cline { 2 - 5 } & 4.337204 & 4.390193 & 4.292229 & 4.153789 \\
\cline { 2 - 5 } & 4.778009 & 4.819037 & 4.72643 & 4.707262 \\
\cline { 2 - 5 } & 4.988728 & 5.00186 & 4.923847 & 4.915685 \\
\hline Cell-20 & 3.431986 & 3.557169 & 3.481558 & 3.465279 \\
\cline { 2 - 5 } & 3.937651 & 4.007779 & 3.995323 & 3.927386 \\
\cline { 2 - 5 } & 4.44955 & 4.458458 & 4.42265 & 4.414143 \\
\cline { 2 - 5 } Fading & 4.690895 & 4.708322 & 4.664203 & 4.654394 \\
\hline Cell-10 & 5 & 6 & 7 & 8 \\
\cline { 2 - 5 } & 3.914978 & 3.880131 & 3.886846 & 3.71114 \\
\cline { 2 - 5 } & 4.346318 & 4.290314 & 4.342645 & 4.121726 \\
\cline { 2 - 5 } & 4.926997 & 4.915923 & 4.898965 & 4.786733 \\
\hline Cell-20 & 3.417669 & 3.439157 & 3.406447 & 3.352868 \\
\cline { 2 - 5 } & 3.933301 & 3.911821 & 3.896017 & 3.857265 \\
\cline { 2 - 5 } & 4.362921 & 4.34666 & 4.289312 & 4.245983 \\
\cline { 2 - 5 } & 4.628451 & 4.620932 & 4.575075 & 4.540827 \\
\hline
\end{tabular}

Table 3 shows the detailed comparative discussion of throughput based on log-normal shadowing fading. Figs. 6 and 7 the shows impact of the transmission power on the achievable throughput at cell-10 and cell-20, where pilot transmission power varies 2 to $20 \mathrm{~dB}$. In figure 6 , throughput at $10 \mathrm{~dB}$ transmission power by our proposed method is 4.69 $\mathrm{bps} / \mathrm{Hz}$, which is $21 \%, 12 \%$, and $4 \%$ more compared to MRT-ZF, WGC-PD, and DPSM. In Fig. 7, throughput at $10 \mathrm{~dB}$ transmission power by our 


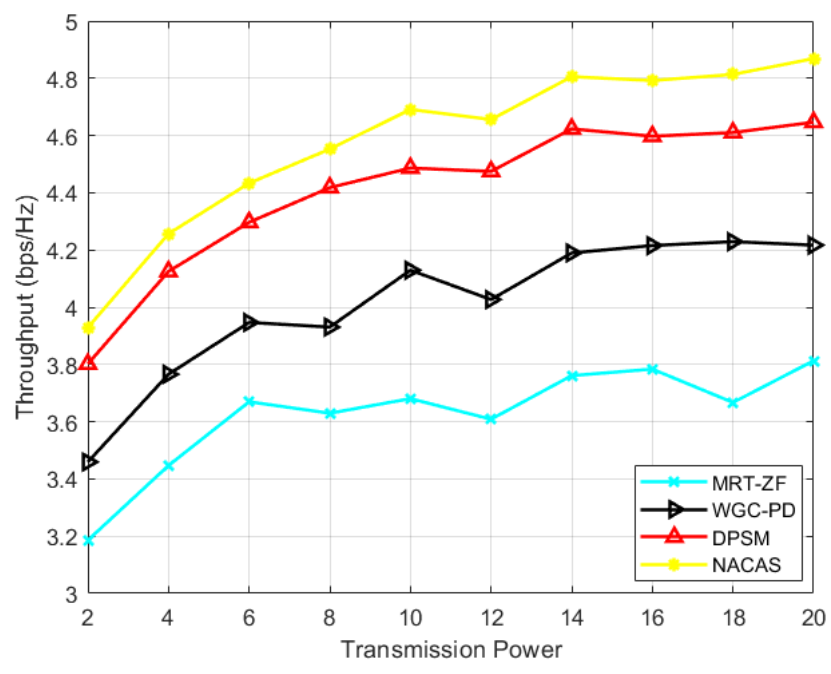

Figure. 5 Impact of the transmission power on the achievable throughput (cell-10)

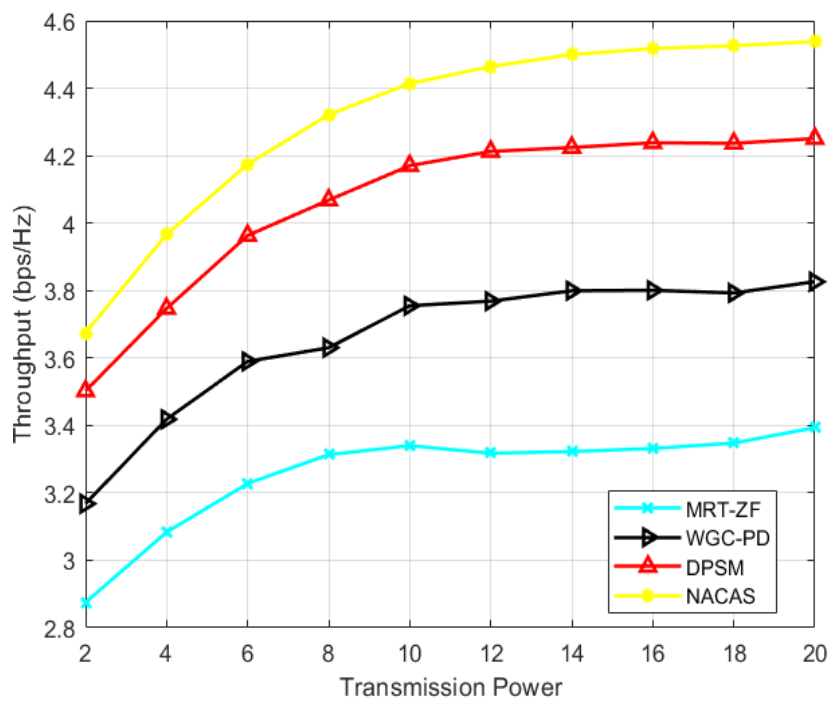

Figure. 6 Impact of the transmission power on the achievable throughput (cell-20)

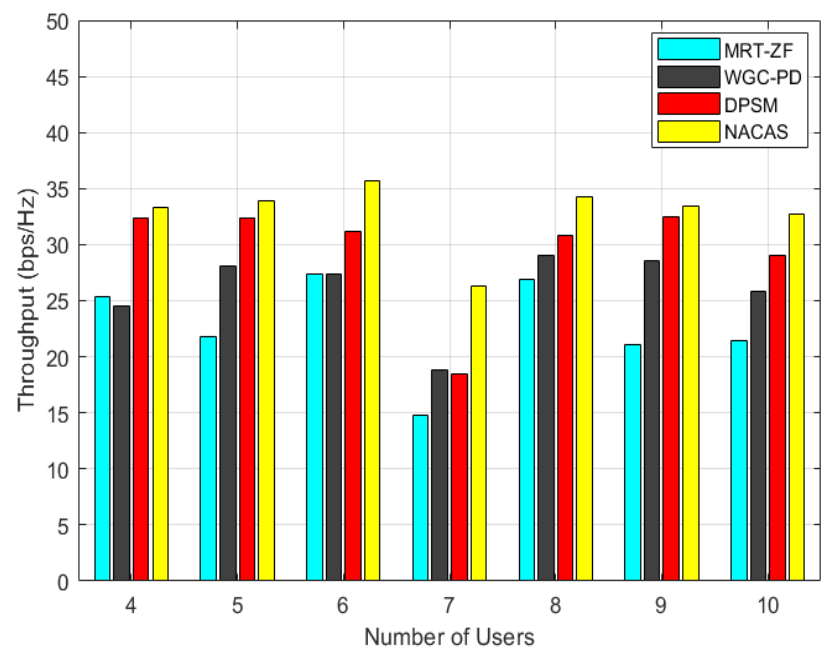

Figure. 7 Impact of the number of users on the achievable throughput (cell-10)
Table 4. Comparative discussion based on transmission

\begin{tabular}{|c|r|r|r|r|r|}
\hline Power & 2 & 4 & 6 & 8 & 10 \\
\hline Cell-10 & 3.186 & 3.447 & 3.67 & 3.63 & 3.681 \\
\cline { 2 - 6 } & 3.459 & 3.766 & 3.947 & 3.931 & 4.129 \\
\cline { 2 - 6 } & 3.802 & 4.125 & 4.297 & 4.418 & 4.486 \\
\cline { 2 - 6 } & 3.929 & 4.257 & 4.433 & 4.553 & 4.691 \\
\hline Cell-20 & 2.875 & 3.083 & 3.228 & 3.313 & 3.34 \\
\cline { 2 - 6 } & 3.168 & 3.418 & 3.59 & 3.63 & 3.755 \\
\cline { 2 - 6 } & 3.502 & 3.747 & 3.963 & 4.068 & 4.17 \\
\cline { 2 - 6 } Power & 3.675 & 3.966 & 4.174 & 4.322 & 4.414 \\
\hline Cell-10 & 3.609 & 3.761 & 3.784 & 3.667 & 3.813 \\
\cline { 2 - 6 } & 4.027 & 4.19 & 4.215 & 4.229 & 4.217 \\
\cline { 2 - 6 } & 4.475 & 4.623 & 4.597 & 4.61 & 4.646 \\
\cline { 2 - 6 } & 4.656 & 4.806 & 4.792 & 4.813 & 4.869 \\
\hline Cell-20 & 3.317 & 3.323 & 3.332 & 3.348 & 3.393 \\
\cline { 2 - 6 } & 3.769 & 3.799 & 3.801 & 3.793 & 3.826 \\
\cline { 2 - 6 } & 4.212 & 4.224 & 4.238 & 4.237 & 4.251 \\
\cline { 2 - 6 } & 4.464 & 4.5 & 4.518 & 4.526 & 4.538 \\
\hline
\end{tabular}

Table 5: Comparative discussion based on number of users

\begin{tabular}{|c|c|c|c|c|}
\hline $\begin{array}{c}\text { No. of } \\
\text { Users }\end{array}$ & 4 & 5 & 6 & 7 \\
\hline Cell-10 & 25.339 & 21.82 & 27.39 & 14.73 \\
\cline { 2 - 5 } & 24.548 & 28.07 & 27.39 & 18.8 \\
\cline { 2 - 5 } & 32.39 & 32.4 & 31.21 & 18.44 \\
\cline { 2 - 5 } & 33.305 & 33.9 & 35.68 & 26.31 \\
\hline Cell-20 & 10.231 & 11.67 & 10.2 & 9.355 \\
\cline { 2 - 5 } & 15.037 & 13.12 & 11.09 & 12.94 \\
\cline { 2 - 5 } & 17.119 & 17.39 & 12.99 & 16.59 \\
\cline { 2 - 5 } Users & 20.092 & 18.7 & 17.51 & 19.16 \\
\hline Cell-10 & 8 & 9 & 10 & \\
\cline { 2 - 5 } & 26.847 & 21.1 & 21.45 & \\
\cline { 2 - 5 } & 28.99 & 28.5 & 25.85 & \\
\cline { 2 - 5 } & 30.835 & 32.5 & 29.08 & \\
\hline Cell-20 & 18.004 & 29.22 & 16.14 & \\
\cline { 2 - 5 } & 23.261 & 34.1 & 23 & \\
\cline { 2 - 5 } & 24.225 & 38.15 & 24.27 & \\
\cline { 2 - 5 } & 26.773 & 38.61 & 27.24 & \\
\hline
\end{tabular}

proposed method is $4.41 \mathrm{bps} / \mathrm{Hz}$, which is $24 \%, 15 \%$, and $5.5 \%$ more compared to MRT-ZF, WGC-PD and DPSM. Table 3 shows the detailed comparative discussion of throughput based on transmission power. Similarly, Fig. 7 shows the significant improvement by the proposed method on the 


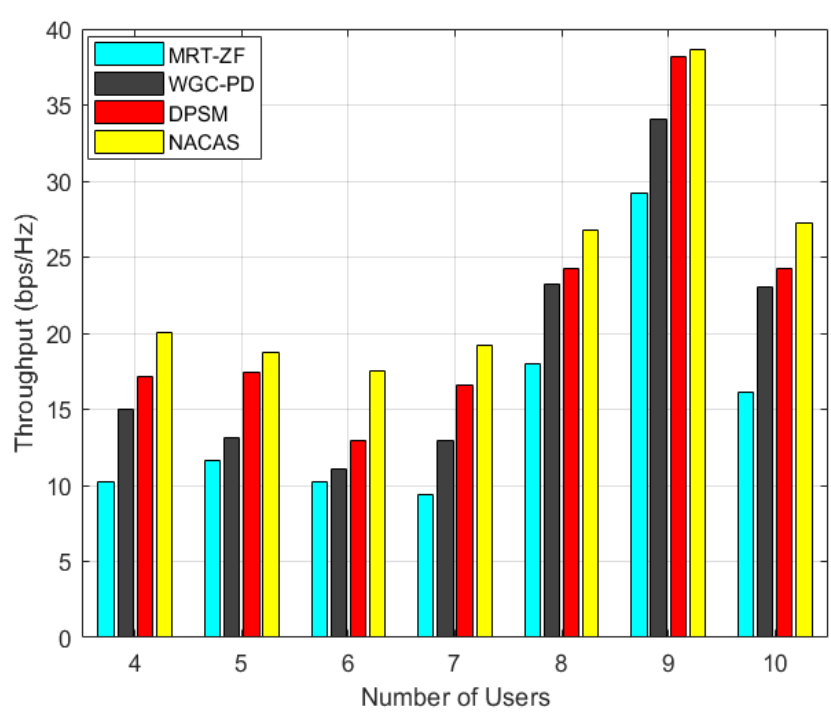

Figure. 8 Impact of the number of users on the achievable throughput (cell-20)

achievable throughput w.r.t. transmission power at cell-20. Detailed comparative discussion on transmission power is shown in Table 4.

The impact of the number of users on the achievable throughput at cell-10 is presented in Fig. 8 , where the number of users varies from 4 to 10.Degradation of throughput is observed at 7 users, while in the rest of the number throughput is almost equal at considered approaches. In Fig. 9, throughput at 9 users by the proposed method is $38.6 \mathrm{bps} / \mathrm{Hz}$, which is $24.3 \%, 11.7 \%$, and $1.2 \%$ more compared to MRT-ZF, WGC-PD, and DPSM. Table 5 shows the detailed comparative discussion of throughput based on the number of users. The above analysis shows that the proposed scheme can improve the system performance effectively.

\section{Conclusion}

The massive-MIMO system contains a various number of users, which makes it difficult to allocate channels. In addition, it involves pilot contamination for getting enhanced spectral efficiency and noise contamination. The communication between BS and user is initiated by the pilot's signal to count the available channels, at the same time there is a chance where a user may communicate to pilots and cause contamination of the channel. In this paper, we majorly focus on channel allocation and decreasing channel contamination for that we have proposed an adaptive channel allocation technique. The performance analysis of our developed scheme is done by varying the number of antennas, log-normal shadowing fading, transmission power, and the number of users concerning throughput. Even our proposed method is compared with other state-of-art techniques, where it shows significant improvement. At this work the analysis is done in up-link transmission, further, we can provide the analysis on Downlink transmission using our proposed method.

\section{Conflicts of interest}

The authors whose names are listed above certify that they have NO affiliations with or involvement in any organization or entity with any financial interest (such as honoraria; educational grants; participation in speakers' bureaus; membership, employment, consultancies and stock ownership), or non-financial interest in the subject matter or materials discussed in this manuscript.

\section{Author contributions}

Both authors contributed to the design and implementation of the research, to the analysis of the results and to the writing of the manuscript.

\section{References}

[1] G. J. Foschini and M. J. Gans, "On limits of wireless communications in a fading environment when using multiple antennas", Wireless Personal Commun., Vol. 6, pp. 311335, 1998.

[2] T. L. Marzetta, "Noncooperative cellular wireless with unlimited numbers of base station antennas", IEEE Trans. Wireless Commun, Vol. 9, pp. 3590-3600, 2010.

[3] K. K. Wong, R. D. Murch, and K. B. Letaief, "A joint-channel diagonalization for multiuser MIMO antenna systems", IEEE Trans. Wireless Commun., Vol. 2, No. 4, pp. 773-786, 2003.

[4] R. Kudo, S. M. D. Armour, J. P. M. Geehan and M. Mizoguchi, "A channel state information feedback method for massive MIMO-OFDM", Journal of Communications and Networks, Vol. 15, No. 4, pp. 352-361, 2013.

[5] Z. Gao, L. Dai, Z. Wang, and S. Chen, "Spatially common sparsity based adaptive channel estimation and feedback for FDD massive MIMO”, IEEE Transactions on Sig. Proc., Vol. 63, No. 23, pp. 6169-6183, 2015.

[6] J. C. Shen, J. Zhang, E. Alsusa, and K. B. Letaief, "Compressed CSI acquisition in FDD massive MIMO: How much training is needed?", IEEE Transactions on Wireless Communications, Vol. 15, No. 6, pp. 4145-4156, 2016.

[7] H. Almosa, R. Shafin, S. Mosleh, Z. Zhou, Y. Li, J. Zhang, and L. Liu, "Downlink channel 
estimation and precoding for FDD 3D Massive MIMO/FD-MIMO systems", In: Proc. of Wireless and Optical Communication Conference (WOCC) 26th. IEEE, pp. 1-4, 2017.

[8] X. Du, J. Tadrous, and A. Sabharwal, "Sequential beamforming for multiuser MIMO with full-duplex training", IEEE Trans. Wireless Commun., Vol. 15, No. 12, pp. 8551-8564, 2016.

[9] Q. Wang and Y. Jing, "Performance analysis and scaling law of MRC/MRT relaying with CSI error in multi-Pair massive MIMO systems", IEEE Trans. Wireless Commun., Vol. 16, No. 9, pp. 5882-5896, 2017.

[10] Y. Jeon, C. Song, S. R. Lee, S. Maeng, J. Jung, and I. Lee, "New beamforming designs for joint spatial division and multiplexing in largescale MISO multi-user systems", IEEE Trans. Wireless Commun., Vol. 16, No. 5, pp. 30293041, 2017.

[11] D. Gesbert, M. Kountouris, R. W. Heath Jr., C. B. Chae, and T. S. alzer, "Shifting the MIMO paradigm", IEEE Sig. Proc. Mag., Vol.24, No.5, pp. 36-46, 2007.

[12] N. Varshney and A. K. Jagannatham, "Cognitive Decode-and-Forward MIMO-RF/FSO Cooperative Relay Networks", IEEE Communications Letters, Vol. 21, No. 4, pp. 893-896, 2017.

[13] I. Kumar, V. Sachan, R. Shankar, and R. K. Mishra, "An investigation of wireless S-DF hybrid satellite terrestrial relaying network over time selective fading channel", Traitement $d u$ Signal (SCIE), Vol. 35, No. 2, pp. 103-120, 2018.

[14] V. Sachan, I. Kumar, R. Shankar, and R. K. Mishra, "Analysis of transmit antenna selection based selective decode forward cooperative communication protocol", Traitement du Signal (SCIE), Vol. 35, No. 1, pp. 47-60, 2018.

[15] V. Sachan, R. Shankar, and R. K. Mishra. "Selective Decode-Forward Cooperative Communication over Nakagami-m Fading Channel with Channel Estimation Error", Journal of Telecommunication, Electronic and Computer Engineering (JTEC), Vol. 9, No. 2-6, pp. 85-90, 2017.

[16] V. Sachan, R. Shankar, I. Kumar, and R. K. Mishra, "Performance Analysis of SM-MIMO System Employing Binary PSK and M'ary PSK Techniques Over Different Fading Channels", Procedia Computer Science, Vol. 152, pp. 323332, 2019.

[17] R. Shankar, I. Kumar, and R. K. Mishra, "Outage Probability Analysis of MIMO-OSTBC Relaying Network Over Nakagamim Fading
Channel Conditions", Traitement $d u$ Signal (SCIE), Vol. 36, No. 1, pp. 59-64, 2019.

[18] R. Shankar, I. Kumar, A. Kumari, K. N. Pandey, and R. K. Mishra, "Pairwise Error Probability Analysis and Optimal Power Allocation for Selective Decode-Forward Protocol Over Nakagami-m Fading Channels", In: Proc. of International Conference on Algorithms, Methodology, Models and Applications in Emerging Technologies (ICAMMAET), Chennai, pp. 1-6, 2017.

[19] Y. Wu, T. Liu, M. Cao, L. Li, and W. Xu, "Pilot contamination reduction in massive MIMO systems based on pilot scheduling", EURASIP Journal on Wireless Communications and Networking, Vol. 1, pp. 21, 2018.

[20] J. Li, D. Wang, P. Zhu, J. Wang, and X. You, "Downlink spectral efficiency of distributed massive MIMO systems with linear beamforming under pilot contamination", IEEE Transactions on Vehicular Technology, Vol. 67, No. 2, pp. 1130-1145, 2018.

[21] M. H. Mazlan, M. Behjati, R. Nordin, and M. Ismail, "Wiener-based smoother and predictor for massive-MIMO downlink system under pilot contamination", Telecommunication Systems, Vol. 67, No. 3, pp. 387-399, 2018.

[22] A. Bulut and A. O. Y1lmaz, "Oversampling in One-Bit Quantized Massive MIMO Systems and Performance Analysis", IEEE Transactions on Wireless Communications, Vol. 12, No. 7, 2018.

[23] S. Park, J. W. Choi, J. Y. Seol, and B. Shim, "Expectation-Maximization-Based Channel Estimation for Multiuser MIMO Systems", IEEE Trans. Commun., Vol. 65, pp. 2397-2410, 2017.

[24] Z. Zhou, J. Fang, L. Yang, H. Li, Z. Chen, and R. S. Blum, "Low-Rank Tensor DecompositionAided Channel Estimation for Millimeter Wave MIMO-OFDM Systems", IEEE J. Sel. Areas Commun., Vol.35, pp. 1524-1538, 2017.

[25] T. Yoo and A. Goldsmith, "Capacity and power allocation for fading MIMO channels with channel estimation error", IEEE Transactions on Information Theory, Vol. 52, No. 5, pp. 22032214, 2006.

[26] R. S. Prabhu and B. Daneshrad, "An EnergyEfficient Water-Filling Algorithm for OFDM Systems", In: Proc. of 2010 IEEE International Conference on Communications, pp. 1-5, 2010.

[27] P. P. Justin and G. R. Krishna, "Power and Channel Allocation on MIMO Systems in Cognitive Radio Network", Wireless Personal Communications Vol. 116, pp. 45-60, 2021. 
[28] J. Hoydis, S. T. Brink, and M. Debbah, "Massive MIMO in the UL/DL of Cellular Networks: How Many Antennas Do We Need?", IEEE Journal on Selected Areas in Communications, Vol. 31, No. 2, pp. 160-171, 2013.

[29] X. Zhu, L. Dai, Z. Wang and X. Wang, "Weighted-Graph-Coloring-Based Pilot Decontamination for Multicell Massive MIMO Systems", in IEEE Transactions on Vehicular Technology, Vol. 66, No. 3, pp. 2829-2834, 2017. 